\title{
VITAMINA C EN LA SALUD Y EN LA ENFERMEDAD
}

\author{
VITAMIN C IN HEALTH AND DISEASE \\ Edwin Rolando Castillo-Velarde $\mathrm{e}^{1, a, b}$
}

\begin{abstract}
RESUMEN
La vitamina $\mathrm{C}$ ha sido ampliamente estudiada en medicina y si bien se reconoció la importancia de su deficiencia con el escorbuto, la optimización de su uso como recurso terapéutico no ha sido incluida en protocolos o guías de práctica clínica. La farmacocinética y biología de la vitamina $\mathrm{C}$ demuestran los efectos sistémicos que posee, y fundamentado en ello, se vienen desarrollado en los últimos años investigaciones que sustenten su uso parenteral en algunas enfermedades. La evidencia disponible señala que el beneficio de su uso no se extiende a varias enfermedades sino a algunas como el cáncer, cuyo reporte preliminar es promisorio, con adecuada tolerabilidad a altas dosis, pero que aún precisa de completar el seguimiento prospectivo de la intervención.
\end{abstract}

Palabras clave: Vitamina C; Cáncer; Alta dosis. (fuente: DeCS BIREME)

\begin{abstract}
Vitamin $C$ has been widely studied in medicine and although the importance of its deficiency with scurvy was recognized, the optimization of its use as a therapeutic resource has not been included in protocols or clinical practice guidelines. The pharmacokinetics and biology of vitamin C indicates the systemic effects it has, and based on it, research has been developed in recent years that supports its parenteral use in some diseases. The available evidence indicates that the benefit of its use does not extend to several diseases but to some such as cancer, whose preliminary report is promising, with adequate tolerability at high doses, but still needs to complete the prospective follow-up of the intervention.
\end{abstract}

Key words: Vitamin C; Cancer; High doses. (source: MeSH NLM)

\section{INTRODUCCIÓN}

El oxígeno como molécula oxidante interviene en la producción de energía, sin embargo, genera también productos intermediarios reactivos o radicales libres que según su grado oxidante pueden dañar a moléculas biológicas como proteínas, lípidos yácidos nucleicos. Este proceso de estrés oxidativo lleva a la producción de especies reactivas de oxígeno como los radicales libres y que, en condiciones fisiológicas, no ocurre en más del $5 \%$ por la regulación endógena antioxidante'. Este nivel oxidativo mínimo tiene importancia funcional, por ejemplo, en el efecto inmune de los neutrófilos, para la actividad de la enzima mieloperoxidasa.
El ambiente es fuente de especies reactivas de oxígeno. En general, las fuentes exógenas están constituidas por diferentes sustancias xenobióticas, que son elementos inorgánicos como productos químicos, de los que se conocen 4 millones de estas sustancias xenobióticas, 63 mil son de uso común y 11 mil pueden ser ingeridos directamente como fármacos o aditivos en los alimentos. Existen otros 50 mil elementos contaminantes en el medio ambiente $^{2}$, por ejemplo, las sustancias químicas derivadas del humo del cigarrillo, en donde se han descrito sustancias radioactivas como el polonio 210, que se vincula al cáncer de pulmón. En cuanto a las

${ }^{1}$ Facultad de Medicina Humana, Universidad Ricardo Palma, Lima-Perú.

a Doctor en Filosofía

b Master en Nutrición Clínica

Citar como: Edwin Rolando Castillo-Velarde. Vitamina C en la salud y en la enfermedad. Rev. Fac. Med. Hum. Octubre 2019; 19(4):95-100. DOI 10.25176/RFMH.v19i4.2351

Artículo publicado por la Revista de la Facultad de Medicina Humana de la Universidad Ricardo Palma. Es un artículo de acceso abierto, distribuído bajo los términos de la Licencia Creative Commons: Creative Commons Attribution 4.0 International, CC BY 4.0 (https://creativecommons.org/licenses/by/4.0/), que permite el uso no comercial, distribución y reproducción en cualquier medio, siempre que la obra original sea debidamente citada. Para uso comercial, por favor póngase en contacto con revista.medicina@urp.pe 
radiaciones, un fumador de 1 paquete y medio por día a lo largo de un año, supone una radiación equivalente a 300 radiografías de tórax ${ }^{3}$, lo cual equivale a 30 milisieverts (mSv), lo que supera las recomendaciones de la Comisión Internacional de Protección Radiológica, que para el público en general, fija un valor máximo de $1 \mathrm{mSv}$ al año4.

La respuesta a estrés oxidante, ocurre por diferentes sustancias endógenas de capacidad antioxidante como el glutatión, la superóxido dismutasa, la catalasa, entre otros, pero también tenemos fuentes de antioxidantes exógenos provenientes de la dieta en donde se incluyen compuestos fenólicos como los flavonoides, carotenoides y vitaminas como la C, D y E.

\section{BIOLOGÍA DE LA VITAMINA C}

La vitamina C, evolutivamente aparece con la capacidad de sintetizar acido ascórbico en los vertebrados terrestres al término de la era paleozoica, como respuesta al aumento dramático del oxígeno atmosférico. Esta crisis tóxica e inusual, llevó a la extinción en masa de los organismos en el periodo pérmico, y solo aquellos tetrápodos que desarrollaron un sistema antioxidante sobrevivieron. Es ahí donde surge la enzima gulonolactona oxidasa, que sintetiza la vitamina C. Sin embargo, el hombre, el mono, algunas especies de cerdos y varias especies de aves, pierden la capacidad de producir esta enzima 5 .

Existen dos importantes formas biológicas de vitamina C, la forma reducida o ácido ascórbico, y la forma oxidada, DHA (ácido dehidroascórbico). La mayor concentración de vitamina $C$ se encuentra a nivel tisular por lo que es importante su transporte. La forma reducida o ácido ascórbico se transporta a nivel intracelular a través de los transportadores SVCTs (Sodium dependent vitamin C transporters). El ácido dehidroascórbico se transporta por los GLUT (transportador de la glucosa) por la semejanza química que existe entre la glucosa $(\mathrm{C} 6 \mathrm{H} 12 \mathrm{O} 6)$ y la vitamina $\mathrm{C}$ (C6H806). La forma oxidada de vitamina C (DHA), una vez que alcanza el intracelular, sufre una reversión espontánea a su forma reducida o ácido ascórbico por la acción del glutatión. Si no se diera este proceso se formarían compuestos inactivos como el ácido ${ }^{2,3}$ diketogulonico y posterior metabolismo a oxalato ${ }^{6,7,8,9}$. En pacientes con hiperoxaluria primaria se restringe el consumo de vitamina $C$ por la posible formación de oxalato en especial si el consumo es excesivo ${ }^{8}$.

Cuando el ácido dehidroascórbico ingresa a nivel mitocondrial, es importante su reducción a ácido ascórbico porque se requiere de mecanismos antioxidantes en respuesta a la fosforilación oxidativa mitocondrial. El daño a nivel del ADN (ácido desoxirribonucleico) mitocondrial frente a este estrés oxidante es 3 a 10 veces mayor que el daño del ADN nuclear ${ }^{10}$.

Un antioxidante, por definición, es aquel que tiene la capacidad de donar electrones al radical libre que se encuentra inestable con el fin de prevenir la oxidación de otros compuestos. Cuando un antioxidante dona sus electrones, se convierte en un radical libre, pero no tiene la capacidad de ser reactivo. En este sentido, la vitamina C luego de donar un electrón, se convierte en el radical ascorbilo (o ácido semidehidroascórbico), pero es relativamente estable y poco reactivo. Luego de la pérdida de su segundo electrón, es cuando se forma el ácido dehidroascórbico. La reducción a ácido ascórbico será la más estable ${ }^{8}$.

La vitamina C dona electrones a 8 diferentes tipos de enzimas, de las cuales 3 participan en la hidroxilación del colágeno (adiciona grupos hidroxilo a aminoácidos prolina y lisina de la molécula del colágeno) incrementando su estabilidad. De allí que lo síntomas del escorbuto se asocien a alteraciones del tejido conectivo, como fragilidad capilar, equimosis, gingivorragias, hemorragias perifoliculares y inadecuada cicatrización de heridas ${ }^{11,8}$. Otras dos enzimas que son reguladas por la vitamina $C$, a través de dos dioxigenasas, intervienen en la síntesis de carnitina, permitiendo el transporte de ácidos grasos para su oxidación. Tres enzimas restantes, participan en la formación de noradrenalina desde dopamina, en agregar grupos amida a hormonas peptídicas y en el metabolismo de la tirosina ${ }^{8}$.

La vitamina $C$ puede reducir diversas sustancias como: 1) compuestos derivados de las especies reactivas de oxígeno (SRO), como el superóxido o radical hidroxilo, y especies reactivas de nitrógeno (SRN), como óxido nítrico, dióxido de nitrógeno y peroxinitrito. 2) compuestos como el radical alfa-tocoferoxil, que se produce cuando un radical libre interacciona con el alfa tocoferol y el LDL (lipoproteína de baja densidad). Este radical se puede reducir nuevamente a alfa tocoferol gracias a la acción del ascorbato, permitiendo su reutilización metabólica como antioxidante. 3) Elementos como el hierro férrico a ferroso, lo que favorece su absorción intestinal ${ }^{12,8}$. 4) compuestos que son reactivos, pero que no son radicales libres, como el ácido hipocloroso, nitrosaminas y el ozono. Se ha demostrado el efecto mutagénico de los derivados de la 
nitrosamina en el cáncer gástrico y que la concentración de vitamina $C$ en el jugo gástrico es 3 veces mayor que la del plasma en personas sanas. Experimentalmente, concentraciones elevadas de vitamina $C$ inducen apoptosis en células tumorales gástricas mediado por la $\mathrm{p}^{38}$ MAP-kinasa (proteína quinasa activada por mitógenos) ${ }^{13}$, sin embargo, no se habían desarrollado ensayos clínicos de intervención que valoren este aspecto. Sí existen estudios retrospectivos que demuestran una asociación de riesgo entre el bajo consumo de vitamina C y cáncer gástrico (OR 0.40, 95\% IC 0.19-0.83) ${ }^{14}$, pero sólo recientemente se ha publicado un reporte preliminar de un ensayo clínico en cáncer gástrico que combina un régimen de quimioterapia con vitamina $C$ endovenoso, cuyos resultados son favorables, pero no es un estudio comparativo ${ }^{15}$.

Por otro lado, la vitamina $C$ puede favorecer reacciones de oxidación como la de Fenton, que ocurre entre la forma libre de metales como hierro o cobre y el ascorbato. Estos metales al unirse al peróxido de hidrogeno, formarán radicales hidroxilos altamente reactivos, y por ello, estos metales no se encuentran en su forma libre porque son captados por la transferrina o ferritina para el hierro, o la ceruloplasmina para el cobre. El ascorbato puede inducir la liberación de hierro desde la ferritina, siendo una estrategia terapéutica utilizada, por ejemplo, en el tratamiento de la anemia refractaria del paciente en hemodiálisis que dispone de gran saturación de hierro, aunque la evidencia todavía es débil por ensayos clínicos escasos ${ }^{16}$.

La vitamina $C$, a nivel tisular se distribuye hasta en un $52 \%$ en el músculo esquelético y $11 \%$ a nivel cerebral ${ }^{17}$. En las células neuronales la vitamina $C$ en su forma biológica de ácido dehidroascórbico puede atravesar la barrera hematoencefálica a través de los receptores GLUT 1. Experimentalmente se ha demostrado que, en el infarto cerebral, la administración endovenosa de DHA produjo una rápida absorción a nivel cerebral con posterior conversión a ácido ascórbico con propiedades neuroprotectoras al reducir el volumen del infarto ${ }^{18,19,20}$.

\section{Dosis}

Actualmente el RDA (recommended dietary allowance) o dosis recomendada de vitamina $C$ es de $90 \mathrm{mg} / \mathrm{dia}$ en hombres adultos y $75 \mathrm{mg} /$ día en mujeres adultas ${ }^{21}$. Establecer el RDA de una vitamina requiere determinar su concentración sérica y tisularfrente a diferentes dosis, conocer su biodisponibilidad, absorción, excreción urinaria y su potencial toxicidad. Las recomendaciones de dosis de vitamina C se establecieron en 1943, describiéndose que una dosis de 60 mg era el doble de la necesaria para prevenir el escorbuto y era el umbral en el que se empezaba a excretarse la vitamina C por orina. Estudios posteriores de farmacocinética, demostraron una baja incidencia de excreción urinaria en dosis de 100 mg, una biodisponibilidad de 100\% en dosis de $200 \mathrm{mg}$ y una saturación completa cuando la dosis alcanza los 1000 mg/dia. En consecuencia, el RDA se incrementó a 90 mg/día, si bien la farmacocinética sustenta un RDA de 200 mg/dia ${ }^{22}$. Debe considerarse que no se trata de establecer el MDA "minimum dietary allowance" o dosis mínima preventiva de la deficiencia, sino de establecer la dosis óptima que puede variar según la condición clínica de cada paciente.

\section{Usos}

a. Respuesta inmune. A nivel de los leucocitos la vitamina $C$ se puede almacenar hasta 100 veces más durante episodios infecciosos, en comparación a los niveles en sangre ${ }^{22}$. Por otro lado, interviene en la quimiotaxis de neutrófilos y monocitos, proliferación de linfocitos y en la actividad de células natural killer ${ }^{23,24}$. Clínicamente, no se ha evidenciado un efecto consistente en la prevención del resfriado común según último reporte de Cochrane ${ }^{25}$; en el caso de la neumonía, Cochrane reporta que la evidencia aun es débil $^{26}$, por ello el soporte de investigación debiera de migrar a otros contextos clínicos pero relacionados a la respuesta inmune.

b.Cáncer. Desde 1976 se había reportado como favorable el uso de dosis altas endovenosa de vitamina $C$ en el manejo de cáncer ${ }^{27}$. Las concentraciones en sangre pueden alcanzar los $21000 \mathrm{uM} / \mathrm{L}$ a una dosis de 60g/día, a diferencia de las concentraciones que se alcanzan por vía oral con la máxima dosis tolerada de 3 gr/día que no excede los 220 uM/L por el límite de la absorción intestinal. Esta diferencia de hasta 95 veces se ha relacionado con un efecto prooxidante caracterizado por la formación de peróxido de hidrogeno, afectando a células tumorales, lo cual se observa desde una concentración de vitamina $C$ de 1000 a 5000 umol/L ${ }^{28,29}$. Por lo tanto, se plantea un efecto dual prooxidante tumoral selectivo en altas dosis y un efecto antioxidante sistémico, según observaciones in vitro $^{30,31}$. El peróxido de hidrogeno es encontrado a nivel de las células tumorales, pero no en sangre, en razón a la carga antioxidante de moléculas como el glutation o la catalasa de los glóbulos rojos, pero que no hay en los tumores ${ }^{32,33}$. El metabolismo glucolítico incrementado de los tumores favorece la captación de ascorbato por la estructura del transportador ya comentada y una 
vez en el interior, el cumulo de SRO induce muerte de las células tumorales ${ }^{9,34,35,36}$. No se dispone de ninguna revisión sistemática sobre la intervención endovenosa de vitamina $C$ puesto que no se habían desarrollaron ensayos clínicos por décadas en razón a una publicación que desestimó su utilidad en 1985, investigación que utilizó la suplementación oral, y que hoy se sabe que no presenta utilidad terapéutica para este grupo de pacientes ${ }^{37}$. En los últimos años sí se han desarrollado ensayos clínicos. Un ensayo de fase I en pacientes con cáncer gástrico o colorrectal metastásico estudió que una dosis de $1.5 \mathrm{~g} / \mathrm{Kg}$ no reportó ninguna toxicidad y que los efectos relacionados a una infusión rápida o por la carga osmótica elevada, tales como, cefalea, aturdimiento, boca seca o molestias gastrointestinales son inusuales, asimismo, no hubo interacción adversa con la quimioterapia. Asimismo, su eficacia preliminar es promisoria con una respuesta parcial del $58.3 \%$ y un control de enfermedad del $95.8 \%$ con un seguimiento de 8.8 meses $^{15}$. Otro ensayo clínico de fase I y II reportó la adecuada tolerancia de la vitamina $C$ en pacientes con cáncer en tratamiento con quimioterapia utilizando una dosis de 43 gramos sin efectos adversos significativos y en algunos casos mejora sintomática, por ejemplo, incremento de la capacidad funcional ${ }^{38}$.

Epigenéticamente, la vitamina $C$ potencia a los inhibidores de la DNA metiltransferasas, por lo que tiene una acción hipometilante, siendo importante por la metilación aberrante del DNA e histonas en el cáncer, asimismo, se propone que la vitamina C, al favorecer la respuesta inmune, puede favorecer que los retrovirus endógenos, los que normalmente forman $9 \%$ del genoma, induzcan la demetilación del DNA, con lo cual se apertura la investigación en la quimioinmunoterapia ${ }^{36}$.

En cuanto a la prevención primaria, los estudios previamente reportados utilizaron la vía oral para la prevención del cáncer sin demostrar ningún beneficio consistente en tumores sólidos como mama, pulmón, colon o cuello uterino ${ }^{39-43}$.

c.Circulación. Considerando que en fumadores una dosis de vitamina C de 2000 mg/día reduce la presencia de marcadores de estrés oxidativo ${ }^{44}$, se considera su posible efecto en el tono vascular ${ }^{17}$, sin embargo, no se han desarrollado estudios controlados para la prevención primaria o secundaria de enfermedades cardiovasculares ${ }^{45}$.

d.Diabetes. En la diabetes mellitus, además de los mecanismos patogénicos vinculados con la glucotoxicidad y lipotoxicidad, tenemos los relacionados al estrés oxidativo. Se reconoce que la glucosa inhibe la captación de ascorbato ${ }^{46}$, por lo que un estado hiperglicémico se podría asociar a un déficit de ascorbato ${ }^{47}$. En condiciones normales la captación de glucosa, a nivel tisular, es preferente sobre el ascorbato. Para mantener la capacidad antioxidante en la sangre, los glóbulos rojos sintetizan una proteína de membrana, la estomatina, la cual permite que el transportador GLUT 1 prefiera el transporte del DHA sobre el de glucosa. Luego se reduciría a su forma de ácido ascórbico para generar su respectivo efecto antioxidante ${ }^{48}$. En el caso de la retinopatía diabética no se dispone de estudios bien diseñados según Cochrane ${ }^{49}$.

\section{DIETA Y VITAMINA C}

Cuando se estableció la recomendación inicial de $60 \mathrm{mg} /$ día de vitamina $C$, se valoraba solo su efecto antiescorbuto y no el efecto antioxidante cuya necesidad puede variar según el turn-over de la vitamina $C$, como en el embarazo o en estrés físico. En el estrés, la vitamina $C$ interviene en la hidroxilación esteroidea suprarrenal, y por ello se observa un aumento en la excreción urinaria de ácido ascórbico. En efecto, el ácido ascórbico fue aislado en 1928 por Szent-Gyorgyi del tejido suprarrenal como ácido hexurónico o factor antiescorbútico ${ }^{24}$. Los niveles más elevados por gramo de tejido de vitamina $C$ se encuentran en la glándula pituitaria y adrenal.

En Estados Unidos el 25\% de hombres y mujeres consumen menos de 60 mg/día de vitamina C. El 10\% de adultos consume menos del $10 \%$. La fuente primaria de vitamina $C$ en la dieta se muestra en la tabla 1, siendo fuente rica la proveniente de frutas cítricas, kiwi, guayaba, camu-camu, papaya, melón, fresa, mango, tomate, jugo de frutas de naranja y uvas; y vegetales como coliflor, brócoli, repollo, berro, espinaca, pimiento y papa. Un consumo de cinco piezas de frutas y vegetales, proporciona una concentración de más de 200 mg/día de vitamina C.

El consumo de esta fuente de nutrientes, además recae en la presencia de otros antioxidantes como flavonol glicósidos y antocianinas. Pacientes fumadores que ingirieron camucamu (Myrciaria dubia) equivalente a una dosis de 1050 mg de vitamina C tuvieron una mayor capacidad antioxidante y antinflamatoria que, si recibían la dosis equivalente de vitamina $C$ en tabletas ${ }^{50}$, lo que reviste en importancia la participación de los otros componentes bioquímicos. 
Tabla1. La fuente primaria de vitamina C en la dieta.

\section{Fuente (porción)}

Camu-camu (100 g)

Guayaba

Melón (1/4)

Toronja

Kiwi (1)

Mango (1 taza, rodajas)

Naranja (1)

Papaya (1 taza, rodajas)

Fresa (1 taza)

Mandarina (1)

Mango

Fuentes de vitamina $\mathrm{C}^{11,17,8}$

\section{CONCLUSIÓN}

Si bien la evidencia no sostiene algún beneficio en algunas enfermedades como algunos procesos infecciosos, tampoco la evidencia es suficientemente consistente por falta de estudios bien diseñados en otros procesos mórbidos como el cáncer en cuanto a su uso parenteral. Esta reportado su tolerabilidad a altas dosis por vía endovenosa y existen reportes que señalan un beneficio sintomático en la salud. El desarrollo de ensayos clínicos debe mantener la plausibilidad científica fundamentado en la biología de la vitamina $C$, distribución tisular y el tipo de transporte celular que posee. El consumo de frutas y verduras representa una recomendación saludable y el entendimiento de los diversos efectos biológicos de la vitamina $C$ señalan la importancia de su consumo.
Contribuciones de autoría: El autor participó en la génesis de la idea, diseño de proyecto, recolección, análisis de la información y preparación del manuscrito del presente trabajo de investigación.

Financiamiento: Autofinanciado.

Conflicto de interés: El autor declara no tener conflicto de interés en la publicación de este artículo.

Recibido: 20 de junio 2019

Aprobado: 25 de agosto 2019

Correspondencia: Edwin Rolando Castillo Velarde

Dirección: Universidad Ricardo Palma, Facultad de Medicina, Lima-Perú

Teléfono: (00511) 3242983

Correo:edwin.castillo@urp.edu.pe

\section{REFERENCIAS BIBLIOGRÁFICAS}

1. Halliwell B, Free radicals, antioxidants, and human disease: curiosity, cause, or consequence?, Lancet. 1994, 10;344(8924):721-4. Disponible en: https://doi.org/10.1016/s0140-6736(94)92211-x

2. Treguerres J. Fisiología Humana. 3 th ed. McGraw Hill, Madrid, 2005. p. 760-775.

3. Cortés R, Radiaciones ionizantes, Investigación y Ciencia, 2011, 416: 8-10. Disponible en: https://www.investigacionyciencia.es/revistas/investigaciony-ciencia/floracin-526/radiaciones-ionizantes-8873

4. Rego B, Humo Radioactivo, Investigación y Ciencia, 2011, 414: 86-88. Disponible en: https://www.investigacionyciencia.es/revistas/investigaciony-ciencia/la-autntica-revolucin-sexual-522/humo-radiactivo-8706

5. Chatterjee I, Evolution and the biosynthesis of ascorbic acid. Science, 1973; 182(118): 1271-2. Disponible en: https://doi.org/10.1126/ science.182.4118.1271

6. Rivas C, Zúñiga F, Vitamin C transporters, J Physiol Biochem, 2008, 64(4): 357 76. Disponible en: https://doi.org/10.1016/B978-0-12-394316-3.00011-9
7. Koshiishi I, Degradation of dehydroascorbate to 2,3-diketogulonate in blood circulation. Biochim Biophys Acta, 1998; 1425(1): 209-14. Disponible en: https://doi.org/10.1016/s0304-4165\%2898\%2900073-7

8. Sebastian P, Katz Arie, Vitamin C as an antioxidant: Evaluation of its role in disease prevention. Journal of American College of Nutrition 2003, 22 (1).1835. Disponible en: https://www.ncbi.nlm.nih.gov/pubmed/12569111

9. Verrax, Calderon, The controversial place of vitamin $C$ in cancer treatment. Biochemical Pharmacology 2008, 76: 1644-52. Disponible en: https://dx.doi. org/10.3389\%2Ffphys.2018.01182

10. Mandl J, Szarka A, Vitamin C: update on physiology and pharmacology, British Journal of Pharmacology, 2009; 157: 1097-110. Disponible en: https:// doi.org/10.1111/j.1476-5381.2009.00282.x

11. Mahan L, Escott-Stump S, Nutrición y Dietoterapia de Krause. 10 th ed. McGraw Hill, Madrid, 2001. p. 109-114.

12. Car A, Frei B, Toward a new recommended dietary allowance for vitamin $C$ based on antioxidant and health effects in humans. Am J Clin Nutr, 1999; 69: 1086-107. Disponible en: https://doi.org/10.1093/ajcn/69.6.1086 
13. Mi $Y$, Kyu $M$, Jung $H$, et al, High concentrations of ascorbic acid induces apoptosis of human gastric cancer cell by p38-MAP kinase-dependent up-regulation of transferrin receptor, Cancer Letters 277 (2009) 48-54. Disponible en: https://doi.org/10.1016/j.canlet.2008.11.020

14. Jenab $M$, Riboli $E$, Ferrari $P$, Plasma and dietary vitamin $C$ levels and risk of gastric cancer in the European Prospective Investigation into Cancer and Nutrition (EPIC-EURGAST), Carcinogenesis 2006; 27 (11): 2250-2257. Disponible en: https://doi.org/10.1093/carcin/bgl096

15. Feng Wang F, He M, Wang Z, et al, Phase I study of high-dose ascorbic acid with mFOLFOX6 or FOLFIRI in patients with metastatic colorectal cancer or gastric cancer, BMC Cancer 2019; 19: 460. Disponible en: https:// doi.org/10.1186/s12885-019-5696-z

16. Tsubakihara Y, Nishi S, Akiba T, et al, 2008 Japanese Society for Dialysis Therapy: Guidelines for Renal Anemia in Chronic Kidney Disease, Therapeutic Apheresis and Dialysis 2010; 14(3):240-275. Disponible en: https://doi.org/10.1111/j.1744-9987.2010.00836.x

17. Gil A, Tratado de Nutrición, Segunda edición. ed. Panamericana, Madrid, 2010. Tomo I, pag: 483-87.

18. Spector R, Nutrient transport systems in brain: 40 years of progress ournal of Neurochemistry. 2009; 111: 315-20. Disponible en: https://doi. org/10.1111/j.1471-4159.2009.06326.x

19. Mack W, Mocco J, A cerebroprotective dose of intravenous citrate/ sorbitol-stabilized Dehydrosacorbic acid is correlated with increased cerebral ascorbic acid and inhibited lipid peroxidation after murine reperfused stroke, Neurosurgery, 2006; 59(2): 383-88. Disponible en: https:// doi.org/10.1227/01.NEU.0000223496.96945.A7

20. Huang J, Agus D, Dehydroascorbic acid, a blood-brain barrie transportable form of vitamin C, mediates potent cerebroprotection in experimental stroke, Proc Natl Acad Sci, 2001; 98(20): 11720-724. . Disponible en: https://doi.org/10.1073/pnas.171325998

21. National Academy of Sciences. Institute of Medicine. Food and Nutrition Board. Dietary Reference Intakes for Vitamin C, Vitamin E, Selenium, and Carotenoids, 2000, National Academy Press, Washington D.C. Pag: 95-185. Disponible en: http://dx.crossref.org/10.17226/9810

22. Levine M, Cantillena C, Vitamin C pharmacokinetics in healthy volunteers: Evidence for a recommended dietary allowance, Proc Natl Acad Sci, 1996 93: 3704-09. . Disponible en: https://doi.org/10.1073/pnas.93.8.3704

23. Wintergerst $E_{\text {, Maggini }} \mathrm{S}$, Immune-Enhancing Role of Vitamin $\mathrm{C}$ and Zinc and Effect on Clinical Conditions. Ann Nutr Metab 2006;50: 85-94. Disponible en: https://doi.org/10.1159/000090495

24. Wintergerst E, Maggini S, Contribution of Selected Vitamins and Trace Elements to Immune Function. Ann Nutr Metab 2007:51: 301-323. Disponible en: https://doi.org/10.1159/000107673

25. Douglas RM, Hemilä H, Chalker E, Treacy B. Vitamin C for preventing and treating the common cold. Cochrane Database Syst Rev. 18 de julio de 2007;(3):CD000980. Disponible en: https://doi.org/10.1002/14651858. CD000980.pub4

26. Hemilä $\mathrm{H}$, Louhiala P. Vitamin $\mathrm{C}$ for preventing and treating pneumonia. Cochrane Database Syst Rev. 8 de agosto de 2013;(8):CD005532. Disponible en: https://doi.org/10.1002/14651858.CD005532.pub3

27. Cameron E, Pauling L, Supplemental ascorbate in the supportive treatment of cancer: Prolongation of survival times in terminal human cancer, Proc. Natl. Acad. Sci. USA 1976; 73(10): 3685-3689. Disponible en https://doi.org/10.1073/pnas.73.10.3685

28. Casciari J, Riordan N, Cytotoxicity of ascorbate, lipoic acid, and other antioxidants in hollow fibre in vitro tumours. British Journa of Cancer, 2001; 84(11): 1544-1550. Disponible en: https://dx.doi. org/10.1054\%2Fbjoc.2001.1814

29. Laurent A, Nicco C, Chéreau C, Controlling tumor growth by modulating endogenous production of reactive oxygen species. Cancer Res 2005; 65: 948-956. Disponible en: https://www.ncbi.nlm.nih.gov/pubmed/15705895

30. Schwartz, The dual roles of nutrients as antioxidants and prooxidants Their effects on tumor cell growth. Journal of Nutrition 1996, 126 1221S-1227S. Disponible en: https://doi.org/10.1093/jn/126.suppl_4.1221S

31. Duarte T, Almeida G, Jones G. Investigation of the role of extracellular $\mathrm{H} 2 \mathrm{O} 2$ and transition metal ions in the genotoxic action of ascorbic acid in cell culture models. Toxicology Letters 2007, 170: 57-65.

32. Chen Q, Graham M, Pharmacologic ascorbic acid concentrations selectively kill cancer cells: Action as a pro-drug to deliver hydrogen peroxide to tissues. Proc Natl Acad Sci, 2005; 102(38):13604-609. . Disponible en: https://doi.org/10.1073/pnas.0506390102

33. Ohno S, Ohno Y. High-dose vitamin C (ascorbic acid) therapy in the treatment of patients with advanced cancer. Anticancer Res, 2009; 29(3): 809-15 . Disponible en: https://www.ncbi.nlm.nih.gov/pubmed/19414313

34. Robert A. Gatenby, Robert J, Why do cancers have high aerobic glycolysis?, Nature reviews cancer 2004, 4(11): 891-9. Disponible en: https:// doi.org/10.1038/nrc1478

35. Vera JC, Rivas C, Hua R, Human, HL-60 myeloid leukemia cells transport dehydroascorbic acid via the glucose transporters and accumulate reduced ascorbic acid. Blood 1994, 84: 1628-34. Disponible en: https://www.ncbi. nlm.nih.gov/pubmed/8068952

36. Gillberg L, Orskov A, Liu M, et al, Vitamin C - a new player in regulation of the cancer epigenome, Seminars in Cancer Biology 2018;51: 59-67. Disponible en: https://doi.org/10.1016/j.semcancer.2017.11.001

37. Moertel CG, Fleming TR, Creagan ET, High-dose vitamin C versus placebo in the treatment of patients with advanced cancer who have had no prior chemotherapy. A randomized double-blind comparison, $\mathrm{N}$ Engl J Med. 1985, 17; 312(3):137-41. Disponible en: https://doi.org/10.1056/ NEJM198501173120301

38. Hoffer J, Robitaille L, Zakarian R, High-Dose Intravenous Vitamin C Combined with Cytotoxic Chemotherapy in Patients with Advanced Cancer: A Phase I-II Clinical Trial L. PLoS One. 2015; 10(4): e0120228. Disponible en: https://doi.org/10.1371/journal.pone.0120228

39. Hu F, Changxing J, Yi W, Retinol, vitamins A, C, and E and breast cance risk: a meta-analysis and meta-regression. Cancer Causes Control 2011, 22: 1383-96. Disponible en: https://doi.org/10.1007/s10552-011-9811-y

40. Greenlee $\mathrm{H}$, Hershman D, Jacobson J, Use of antioxidant supplements during breast cancer treatment: a comprehensive review. Breast Cancer Res Treat 2009, 115: 437-452. Disponible en: https://doi.org/10.1007/s10549008-0193-0

41. Papaioannou D, Cooper K, Carroll C, Antioxidants in the chemoprevention of colorectal cancer and colorectal adenomas in the general population: a systematic review and meta-analysis. Colorectal Dis 2011; 13(10): 1085-99. Disponible en: https://doi.org/10.1111/j.1463-1318.2010.02289.x

42. Myung S-K, Ju W, Kim SC, Vitamin or antioxidant intake (or serum level) and risk of cervical neoplasm: a meta analysis. International Journal of Obstetrics and Gynaecology 2011; 118(11): 1285-91. Disponible en: https:// doi.org/10.1371/journal.pone.0183395

43. Caraballoso M, Sacristan M, Serra $C$, et al. Drugs for preventing lung cancer in healthy people. Cochrane Database Syst Rev 2009 [CD002141]. Disponible en: https://doi.org/10.1002/14651858.CD002141

44. Murdeach $\mathrm{R}$, Modulation of oxidant stress in vivo in chronic cigarette smokers. Circulation, 1996; 94(1): 19-25. Disponible en: https://doi. org/10.1161/01.CIR.94.1.19

45. Bjelakovic G, Nikolova D, Gluud LL. Antioxidant supplements for prevention of mortality in healthy participants and patients with various diseases. Cochrane Database Syst Rev 2012 [CD007176]. Disponible en: https://doi.org/10.1002/14651858.CD007176.pub2

46. Steven C. Rumsey, Oran K, Glucose transporter isoforms GLUT1 and GLUT3 transport dehydroascorbic acid, The journal of Biological Chemistry, 1997; 272(30): 18982-989. Disponible en: https://doi.org/10.1074/ jbc.272.30.18982

47. Price K, Price S, Hyperglicemia induced latent scurvy and atherosclerosis: The Scorbutic metaplasia Hypothesis, Medical Hypotheses, 1996; 46: 119 29. Disponible en: https://doi.org/10.1016/s0306-9877(96)90011-0

48. Montel-Hagen A, Kinet S, Erythrocite Glut 1 triggers Dehydroascorbic acid uptake in mammals unable to synthesize vitamin C, Cell, 2008; 132(21): 1039-48. Disponible en: https://doi.org/10.1016/j.cell.2008.01.042

49. Lopes de Jesus C, Atallah A, Valente O. Vitamin C and superoxide dismutase (SOD) for diabetic retinopathy. Cochrane Database Syst Rev 2009 [CD006695]. Disponible en: https://doi.org/10.1002/14651858.CD006695. pub2

50. Inoue T, Komoda H, Tropical fruit camu-camu (Myrciaria dubia) has anti-oxidative and anti-inflammatory properties. Journal of Cardiology. October 2008; 52, Issue 2: 127-132. Disponible en: https://doi.org/10.1016/j. jjcc.2008.06.004. 